\title{
COLOR VARIABILITY OF HBC 722 IN THE POST-OUTBURST PHASES
}

\author{
Giseon Baek ${ }^{1}$, Soojong PaK ${ }^{1}$, Joel D. Green ${ }^{2}$, Stefano Meschiari ${ }^{2}$, Jeong-Eun Lee ${ }^{1}$, \\ Yiseul Jeon ${ }^{3}$, Changsu Choi $^{3}$, Myungshin Im ${ }^{3}$, Hyun-Il Sung ${ }^{4}$, and Won-Kee Park ${ }^{4}$ \\ ${ }^{1}$ School of Space Research and Institute of Natural Sciences, Kyung Hee University, 1732 Deogyeong-daero, Giheung-gu, \\ Yongin-si, Gyeonggi-do 446-701, Korea \\ ${ }^{2}$ Department of Astronomy, University of Texas at Austin, Austin, TX 78712, USA \\ ${ }^{3}$ CEOU/Department of Physics \& Astronomy, Seoul National University, 599 Gwanak-ro, Gwanak-gu, Seoul 151-742, Korea \\ ${ }^{4}$ Korea Astronomy and Space Science Institute, Daejeon 305-348, Korea \\ Received 2014 June 6; accepted 2014 September 30; published 2015 January 27
}

\begin{abstract}
We carried out photometric observations for HBC 722 in the Sloan Digital Sky Survey $r, i$, and $z$ bands from 2011 April to 2013 May with the Camera for QUasars in EArly uNiverse attached to the $2.1 \mathrm{~m}$ Otto Struve telescope at McDonald Observatory. The post-outburst phenomena were classified into five phases according to not only brightness but also color variations, which might be caused by physical changes in the emitting regions of optical and near-infrared bands. A series of spectral energy distributions (SEDs) is presented to support color variations and track the time evolution of the SED in optical/near-infrared bands after the outburst. Given two years of data, possible periodicities of $r, i$, and $z$ bands were checked. We found three families of signals around $\sim 6, \sim 10$, and $\sim 1$ days in three bands, which is broadly consistent with Green et al. We also examined short-term variability (intraday and day scales) to search for evidences of flickering by using the micro-variability method. We found clear signs of day scale variability and weak indications of intra-day scale fluctuations, which implies that the flickering event occurs in HBC 722 after outburst.
\end{abstract}

Key words: accretion, accretion disks - stars: formation - stars: individual (HBC 722) - stars: pre-main sequence stars: variables: T Tauri, Herbig Ae/Be - techniques: photometric

Supporting material: data behind figures

\section{INTRODUCTION}

FU Orionis type objects (FUors) are a group of pre-main sequence objects showing a long-lived outburst in optical bands (Hartmann \& Kenyon 1996). A prototype of this group, FU Orionis, flared up by 6 mag in the $B$ band for a few months in 1936. Since then, FU Orionis has stayed bright for $\sim 80$ years and dimmed only 0.015 mag per year (Kenyon et al. 2000).

A dozen FUors have been discovered with analogous photometric features. They are located in an active star-forming region and show a rapid increase of optical brightness (3-5 mag) within a few months. A ring-shaped asymmetric reflection nebula appears after outburst (Goodrich 1987). They are expected to have a decay time of 10-100 years (Bell \& Lin 1994) although there are small differences in each source (Hartmann \& Kenyon 1996). Another dozen of FUor-like objects have been found via spectral diagnosis. They have an F-G supergiant spectum in optical and a $\mathrm{K}-\mathrm{M}$ giant-supergiant spectrum in near-infrared wavelength region. In addition, they show double-peaked line profiles and P Cygni profiles in $\mathrm{H} \alpha$, and infrared excess in spectral energy distribution (SED; Weintraub et al. 1991; Lee et al. 2011).

FU Orionis type outburst phenomena have been interpreted as a sudden increase in the accretion rate by a factor of 100-1000 as compared with that in the quiescent state. Throughout an entire outburst event, $\sim 0.01 \quad M_{\odot}$ of disk material is supplied to the central star (Bell \& Lin 1994; Hartmann \& Kenyon 1996). In this picture, material reaching the innermost part of the circumstellar disk is dumped into the central star. This process in FUors might give rise to a detectable sign of accretion and material dumping at the inner edge of the disk, often referred to as "flickering." It can also cause inhomogeneities such as cool or hot spots on the stellar surface or disk instability, which are believed to be the reasons of periodic or sporadic variabilities in hour-day timescales.

HBC 722 (also known as LkH $\alpha 188$ G4, PTF 10qpf, and V2493 Cyg) is located in the dark cloud region, named the "Gulf of Mexico," in the southern part of the North America/ Pelican Nebula Complex at a distance of 520 pc (Laugalys et al. 2006). HBC 722 is the second FUor with a wellcharacterized pre-outburst optical spectrum. Before the outburst, the source had characteristics of classical $\mathrm{T}$ Tauri star with a mass of $\sim 0.5 M_{\odot}$, bolometric luminosity of $0.85 L_{\odot}$, visual extinction of $3.4 \mathrm{mag}$, and a small amount of variability generally seen in Class II young stellar objects (YSOs; Semkov et al. 2010; Miller et al. 2011; Kóspál et al. 2011). Its prominent $\mathrm{H} \alpha$ features with equivalent width of $100 \mathrm{~nm}$ imply that the accretion activity was high even in the quiescent state (Cohen \& Kuhi 1979).

In 2010 July, HBC 722 produced a large amplitude optical outburst over a few months $(\Delta V=4.7 \mathrm{mag})$, and was classified as a FUor (Semkov et al. 2010; Miller et al. 2011). After the peak of 2010 September, it darkened by about $1.5 \mathrm{mag}$ in $V$ for six months, which is uncharacteristic of FUors. Kóspál et al. (2011) suggested that, with this rate of decline, HBC 722 could return to the quiescent state in a year. They claimed that it was necessary to reconsider the classification of the source as a FUor, and consider another category of flaring YSO with a shorter outburst duration period, like EXors. They also calculated some properties of HBC 722 after the outburst. They derived the accretion rate of $10^{-6} M_{\odot}$ year $^{-1}$ and a bolometric luminosity of $8-12 L_{\odot}$ assuming the mass of $0.5 M_{\odot}$ and radius of $3 R_{\odot}$. During the outburst, the luminosity rose roughly by a factor of 10 compared with the quiescence luminosity $\left(0.85 L_{\odot}\right)$, which is 
somewhat smaller than classical FUors whose luminosity rises by a factor of 10-100, although the source often shows typical spectra of FUors (Audard et al. 2014).

Since then, however, HBC 722 remained in a relatively constant status with small fluctuations, maintaining a level brighter than that in the quiescent state by $3.3 \mathrm{mag}(V)$ for a few months and then started to re-brighten (Semkov et al. 2012a; Green et al. 2013). According to a recent study, the source has gradually regained about $1.5 \mathrm{mag}$ in $V$ band over two years. Thus, HBC 722 can be classified as a FUor (Audard et al. 2014). Meanwhile, the color also has slightly changed during the re-brightening state. It became bluer by 0.2 mag in $R-I$ color compared with the constant status (Semkov et al. 2014).

In this paper, we present the results of high cadence photometric observations from 2011 April to 2013 May. This observation includes the re-brightening state in optical/nearinfrared wavelengths to track the re-increase of the accretion rate of HBC 722. We trace variabilities of HBC 722 in multiple timescales (year, day, and intra-day) and try to find evidence of flickering. In Section 2, the details of observation strategies and data reduction techniques are described. We analyze behaviors of HBC 722 by using light curves, color curves, colormagnitude diagrams, and color-color diagrams in Section 3. In Section 4 , we discuss the time evolution of SEDs after outburst and diagnosis of flickering with day variability and intra-day variability (IDV) checks. Finally, we summarize our conclusions in Section 5. In this study, we use the $A B$ magnitude system. Also, we deal with "long term" as year or longer timescales and "short term" as day or intra-day timescales.

\section{OBSERVATIONS AND DATA REDUCTION}

We carried out photometric observations of HBC 722 using the Camera for QUasars in EArly uNiverse (CQUEAN) attached to the $2.1 \mathrm{~m}$ Otto Struve telescope at the McDonald Observatory (Kim et al. 2011; Park et al. 2012). Using a $1024 \times 1024$ pixel deep-depletion CCD chip, CQUEAN has a $4.7 \times 4.7$ field of view with a custom-made focal reducer (Lim et al. 2013). We obtained minute scale cadence images in the $r$, $i$, and $z$ bands in the Sloan Digital Sky Survey (SDSS) filter system (Fukugita et al. 1996) for 60 nights from 2011 April to 2013 May. To see the short-scale behavior of HBC 722, we performed continuous time series monitoring observations for $2-8$ hours in 18 nights. The observation field is shown in Green et al. (2013). The log of observations are listed in Table 1.

The images were reduced with the $\mathrm{IRAF}^{5} / \mathrm{CCDRED}$ packages. Since most of the images were exposed for less than 30 s, dark subtraction was unnecessary except for the 2011 December $r$ band images. Aperture photometry was conducted by using Source Extractor (Bertin \& Arnouts 1996). We set the aperture size with three times of FWHM of seeing of each night. Errors include Poisson errors, sky background fluctuations, and subtraction errors for differential photometry. For the nightly averaged points, errors are denoted by the standard deviation of a comparison star in a given night. Typical averaged error value is $\leqslant 0.01 \mathrm{mag}$.

Since HBC 722 is located in an active star-forming region, most of the surrounding objects in our HBC 722 field are likely

\footnotetext{
5 IRAF is distributed by the National Optical Astronomy Observatory, which is operated by the Association of Universities for Research in Astronomy, Inc. under cooperative agreement with the National Science Foundation.
}

to be YSOs, which could show small amplitude variabilities (Semkov et al. 2010; Green et al. 2013). Thus for the differential photometry, we had to carefully select comparison stars in the field. We carried out variability checks on the background objects by repetitive differential photometry, paring two candidates. Finally C7 and C4 (see Table 2 for details) were chosen as a comparison star and check star among 12 selections. Semkov et al. (2010) showed a comparison sequence for HBC 722 field, which consists of 15 objects in the $B V R I$ bands with care for low amplitude variability. In their work, $\mathrm{C} 4$ and $\mathrm{C} 7$ were used as photometric standards and labeled as $\mathrm{A}$ and $\mathrm{C}$, respectively, according to their convention. We present SEDs of two objects in Figure 1. We took $u$ and $g$ bands data from the SDSS database and $r, i$, and $z$ band data from this work. We also took magnitudes from the 2MASS point source catalog (Skrutskie et al. 2006) for the $J, H$, and $K s$ bands, and the Wide-field Infrared Survey Explorer (WISE) source catalog for 3.4, $4.6 \mu \mathrm{m}$ data (Wright et al. 2010). We converted magnitudes of 2MASS and WISE from the Vega to AB magnitude system. In optical/ near-infrared, both the comparison and the check star do not show any special features but have blackbody-like spectra. We fit SEDs assuming a single temperature blackbody and obtained approximate temperature of 3400-3500 K (spectral type M2M3) and 3500-3600 K (spectral type M3-M4) for C4 and C7, respectively.

Flux calibration was conducted using SDSS standard stars from Smith et al. (2002), taken in a photometric night during the 2012 June observing run. We considered only the zero point and airmass terms of the standard calibration formula, since secondary and higher order terms were small enough to be ignored in the flux calibration.

\section{RESULTS}

\subsection{Monitoring Brightness and Color Variabilities}

We observed HBC 722 from 2011 April to 2013 May, during constant and re-brightening states of the object. The upper part of Figure 2 shows light curves for our observations in the $r, i$ and $z$ bands and the Johnson-Cousins $R$ and $I$ band literature data for comparison (Semkov et al. 2012a, 2012b, 2014). We converted the magnitudes of the $R$ and $I$ bands from the Vega to AB magnitude system using Blanton \& Roweis (2007). Our observational and archival data are fairly consistent in terms of monotonic behaviors of brightness, although there are small offsets of magnitude due to differences in the filter system. From 2011 April to 2013 May, HBC 722 brightened $1.8 \mathrm{mag}$ in the $r$ band and $1.6 \mathrm{mag}$ in the $i$ and $z$ bands including smaller scale variations. For comparison, the $R$ and $I$ bands from Semkov et al. (2012a, 2014) got brighter by 1.73 and $1.55 \mathrm{mag}$ in the same period. The bottom part of Figure 2 presents long-term color curves. At the same time, color became bluer about $0.18 \mathrm{mag}$ in $r-i$ and $0.1 \mathrm{mag}$ in $i-z$. $R-I$ color also became bluer by $0.18 \mathrm{mag}$, and from the reddest point, it differed by $0.25 \mathrm{mag}$.

We divide outburst stages of HBC 722 until 2013 May into five phases according to its brightness and color changes (see Table 3 and Figure 2). Phase 1 is from the beginning of the outburst to the first brightness peak in 2010 September. The brightness rapidly increased and color became bluer in phase 1 . Phase 2 is from 2010 September to 2011 February, during which the brightness became fainter and color became redder. These two phases are presented in Semkov et al. (2010) and 
Table 1

Observing Log

\begin{tabular}{|c|c|c|c|c|c|}
\hline \multirow{2}{*}{$\begin{array}{l}\text { Date }^{\text {a }} \\
\text { yyyy-mm-dd }\end{array}$} & \multirow{2}{*}{$\begin{array}{c}\text { Time }^{\mathrm{a}} \\
\text { hh:mm:dd }\end{array}$} & \multirow{2}{*}{$\begin{array}{c}\text { JD } \\
(+2450000)\end{array}$} & \multicolumn{3}{|c|}{ Exposure Time $\times$ Number of Frames ${ }^{b}$} \\
\hline & & & $r$ & $i$ & $z$ \\
\hline 2011-04-26 & $10: 51: 58$ & 5677.9528 & $20 \times 24$ & $20 \times 24$ & $20 \times 24$ \\
\hline 2011-04-30 & $10: 59: 49$ & 5681.9582 & $15 \times 30$ & $15 \times 30$ & $15 \times 30$ \\
\hline 2011-07-05 & $5: 44: 11$ & 5747.7390 & $10 \times 311$ & $10 \times 311$ & $10 \times 311$ \\
\hline 2011-07-06 & $8: 23: 28$ & 5748.8496 & $10 \times 5$ & $10 \times 5$ & $10 \times 5$ \\
\hline 2011-07-07 & $8: 28: 53$ & 5749.8534 & $10 \times 5$ & $10 \times 5$ & $10 \times 5$ \\
\hline 2011-07-08 & $8: 20: 31$ & 5750.8476 & $10 \times 5$ & $10 \times 5$ & $10 \times 5$ \\
\hline 2011-07-09 & 8:06:10 & 5751.8376 & $10 \times 5$ & $10 \times 5$ & $10 \times 5$ \\
\hline 2011-07-11 & $7: 32: 59$ & 5753.8146 & $10 \times 47$ & $10 \times 47$ & $10 \times 47$ \\
\hline 2011-08-19 & 8:33:37 & 5792.8567 & $20 \times 25$ & $10 \times 25$ & $10 \times 25$ \\
\hline $2011-08-21$ & $6: 24: 41$ & 5794.7671 & $20 \times 15$ & $10 \times 15$ & $10 \times 15$ \\
\hline $2011-08-22$ & $6: 39: 34$ & 5795.7775 & $20 \times 15$ & $10 \times 15$ & $10 \times 15$ \\
\hline $2011-08-23$ & $4: 38: 42$ & 5796.6935 & $20 \times 15$ & $10 \times 15$ & $10 \times 15$ \\
\hline $2011-08-24$ & $2: 35: 07$ & 5797.6077 & $20 \times 1125$ & $\ldots$ & $\ldots$ \\
\hline $2011-08-25$ & $4: 37: 44$ & 5798.6929 & $20 \times 15$ & $10 \times 15$ & $10 \times 15$ \\
\hline $2011-08-26$ & $5: 12: 55$ & 5799.7173 & $20 \times 12$ & $10 \times 12$ & $10 \times 12$ \\
\hline $2011-08-27$ & $5: 30: 30$ & 5800.7295 & $20 \times 15$ & $10 \times 15$ & $10 \times 15$ \\
\hline $2011-08-28$ & $4: 55: 42$ & 5801.7054 & $20 \times 15$ & $10 \times 15$ & $10 \times 15$ \\
\hline $2011-08-29$ & $5: 17: 58$ & 5802.7208 & $20 \times 15$ & $10 \times 15$ & $10 \times 15$ \\
\hline $2011-08-30$ & 5:14:34 & 5803.7185 & $20 \times 10$ & $10 \times 10$ & $10 \times 10$ \\
\hline $2011-08-31$ & $3: 34: 55$ & 5804.6493 & $20 \times 791$ & $\ldots$ & $\ldots$ \\
\hline 2011-09-01 & $6: 19: 49$ & 5805.7638 & $30 \times 14$ & $15 \times 2,10 \times 12$ & $10 \times 14$ \\
\hline $2011-10-30$ & $3: 12: 20$ & 5864.6336 & $20 \times 6$ & $10 \times 6$ & $10 \times 6$ \\
\hline 2011-10-31 & $2: 29: 53$ & 5865.6041 & $20 \times 15$ & $10 \times 15$ & $10 \times 15$ \\
\hline 2011-11-01 & $2: 12: 01$ & 5866.5917 & $20 \times 15$ & $10 \times 15$ & $10 \times 15$ \\
\hline 2011-11-02 & 1:05:49 & 5867.5457 & $20 \times 12,30 \times 199$ & $10 \times 211$ & $10 \times 211$ \\
\hline 2011-11-04 & 1:07:04 & 5869.5466 & $30 \times 116$ & $10 \times 116$ & $10 \times 116$ \\
\hline $2011-11-05$ & $2: 14: 58$ & 5870.5937 & $30 \times 14$ & $10 \times 14$ & $10 \times 14$ \\
\hline 2011-11-07 & $0: 47: 35$ & 5872.5330 & $30 \times 15$ & $10 \times 15$ & $10 \times 15$ \\
\hline 2011-11-08 & $1: 31: 37$ & 5873.5636 & $30 \times 3,60 \times 10$ & $10 \times 3,20 \times 10$ & $10 \times 3,20 \times 10$ \\
\hline 2011-11-09 & $0: 48: 21$ & 5874.5336 & $30 \times 179,60 \times 27$ & $10 \times 179,20 \times 27$ & $10 \times 179,20 \times 27$ \\
\hline $2011-12-14$ & 2:09:20 & 5909.5898 & $90 \times 3$ & $30 \times 3$ & $30 \times 3$ \\
\hline $2011-12-16$ & $1: 16: 41$ & 5911.5533 & $90 \times 5$ & $20 \times 5$ & $30 \times 5$ \\
\hline $2012-05-27$ & $8: 28: 31$ & 6074.8531 & $20 \times 45,30 \times 83$ & $10 \times 128$ & $10 \times 128$ \\
\hline 2012-05-30 & $7: 24: 56$ & 6077.8090 & $15 \times 300$ & $4 \times 300$ & $4 \times 300$ \\
\hline 2012-05-31 & $9: 51: 59$ & 6078.9111 & $10 \times 3$ & $5 \times 3$ & $5 \times 3$ \\
\hline $2012-06-26$ & $7: 54: 30$ & 6104.8295 & $15 \times 30$ & $4 \times 30$ & $4 \times 30$ \\
\hline $2012-06-27$ & $6: 58: 05$ & 6105.7903 & $15 \times 29$ & $4 \times 29$ & $4 \times 29$ \\
\hline $2012-06-28$ & $5: 09: 22$ & 6106.7148 & $15 \times 222,20 \times 177$ & $4 \times 222,6 \times 177$ & $4 \times 222,6 \times 177$ \\
\hline $2012-06-29$ & $5: 43: 15$ & 6107.7384 & $15 \times 30$ & $4 \times 30$ & $4 \times 30$ \\
\hline $2012-06-30$ & $5: 29: 25$ & 6108.7288 & $30 \times 30$ & $8 \times 30$ & $8 \times 30$ \\
\hline $2012-07-01$ & $7: 10: 07$ & 6109.7987 & $30 \times 182$ & $8 \times 182$ & $8 \times 182$ \\
\hline 2012-07-02 & $6: 33: 15$ & 6110.7731 & $15 \times 3,30 \times 29$ & $4 \times 3,8 \times 29$ & $4 \times 3,8 \times 29$ \\
\hline 2012-09-01 & $2: 58: 50$ & 6171.6242 & $15 \times 27,20 \times 24,30 \times 277$ & $4 \times 328$ & $4 \times 328$ \\
\hline 2012-09-02 & $2: 51: 35$ & 6172.6192 & $30 \times 77,10 \times 259,15 \times 78,20 \times 78$ & $4 \times 414,6 \times 78$ & $4 \times 336,6 \times 156$ \\
\hline $2012-09-03$ & $4: 44: 14$ & 6173.6974 & $20 \times 198,30 \times 21$ & $4 \times 150,5 \times 48,8 \times 21$ & $5 \times 150,6 \times 48,8 \times 3,10 \times 18$ \\
\hline 2012-09-04 & 8:00:18 & 6174.8335 & $15 \times 14$ & $4 \times 14$ & $4 \times 14$ \\
\hline 2012-09-05 & $7: 14: 20$ & 6175.8016 & $10 \times 38$ & $4 \times 38$ & $5 \times 38$ \\
\hline 2012-09-06 & $3: 37: 17$ & 6176.6509 & $30 \times 23$ & $8 \times 23$ & $10 \times 23$ \\
\hline 2012-09-07 & $2: 35: 53$ & 6177.6083 & $10 \times 31$ & $4 \times 31$ & $6 \times 31$ \\
\hline 2012-09-09 & $3: 18: 30$ & 6179.6379 & $15 \times 162,30 \times 15$ & $4 \times 162,8 \times 15$ & $6 \times 162,12 \times 15$ \\
\hline 2012-09-10 & $2: 49: 43$ & 6180.6179 & $10 \times 22$ & $4 \times 22$ & $6 \times 22$ \\
\hline 2012-09-11 & $4: 48: 35$ & 6181.7004 & $10 \times 24$ & $4 \times 24$ & $6 \times 24$ \\
\hline $2012-09-12$ & $6: 07: 20$ & 6182.7551 & $30 \times 5$ & $8 \times 5$ & $12 \times 5$ \\
\hline $2012-11-23$ & $2: 00: 33$ & 6254.5837 & $30 \times 10$ & $5 \times 10$ & $5 \times 10$ \\
\hline $2012-11-24$ & $1: 52: 13$ & 6255.5779 & $20 \times 30$ & $5 \times 30$ & $5 \times 30$ \\
\hline $2012-11-26$ & $1: 45: 33$ & 6257.5733 & $20 \times 53$ & $5 \times 53$ & $5 \times 53$ \\
\hline 2013-04-30 & 9:03:50 & 6412.8777 & $15 \times 162$ & $4 \times 162$ & $4 \times 162$ \\
\hline 2013-05-01 & $9: 27: 04$ & 6413.8938 & $15 \times 84,10 \times 60$ & $4 \times 144$ & $4 \times 144$ \\
\hline 2013-05-04 & $8: 45: 14$ & 6416.8648 & $15 \times 134,30 \times 9$ & $4 \times 134,8 \times 9$ & $4 \times 134,10 \times 9$ \\
\hline $2013-05-05$ & $8: 30: 23$ & 6417.8544 & $15 \times 150$ & $4 \times 150$ & $4 \times 150$ \\
\hline
\end{tabular}

Note. $r$ band data from 2011 April to 2012 July is mentioned in Green et al. (2013).

${ }^{a}$ Date and time in UT. Time refers when observation started.

${ }^{\mathrm{b}}$ Exposure time per frame in units of second. 
Table 2

Target List

\begin{tabular}{|c|c|c|c|c|c|c|c|}
\hline \multirow[b]{2}{*}{ Target } & \multirow[b]{2}{*}{ USNO-B $1.0^{\mathrm{a}} \mathrm{ID}$} & \multirow[b]{2}{*}{ R.A. (2000) } & \multirow[b]{2}{*}{ Decl. (2000) } & \multicolumn{3}{|c|}{ AB Magnitude } & \multirow[b]{2}{*}{ Spectral Type } \\
\hline & & & & $r$ & $i$ & $z$ & \\
\hline HBC 722 & $1338-0391463$ & 20:58:17.0 & $+43: 53: 42.9$ & $14.3 \sim 12.5^{\mathrm{b}}$ & $13.4 \sim 11.8^{\mathrm{b}}$ & $12.8 \sim 11.2^{\mathrm{b}}$ & Late $\mathrm{K}-\mathrm{M}^{\mathrm{c}}$ \\
\hline $\mathrm{C} 4^{\mathrm{d}}$ & $1338-0391536$ & $20: 58: 30.0$ & $+43: 52: 23.8$ & 14.31 & 13.80 & 13.50 & M2-M3 \\
\hline $\mathrm{C} 7$ & $1338-0391522$ & $20: 58: 26.3$ & $+43: 52: 23.5$ & 15.15 & 14.53 & 14.20 & M3-M4 \\
\hline
\end{tabular}

a http://www.nofs.navy.mil/data/fchpix/

${ }^{\mathrm{b}}$ Brightness changes in our observed period (2011 April-2013 May) are shown.

${ }^{c}$ Miller et al. (2011).

${ }^{\mathrm{d}}$ Coordinate and $r$ mag from Green et al. (2013) are quoted.

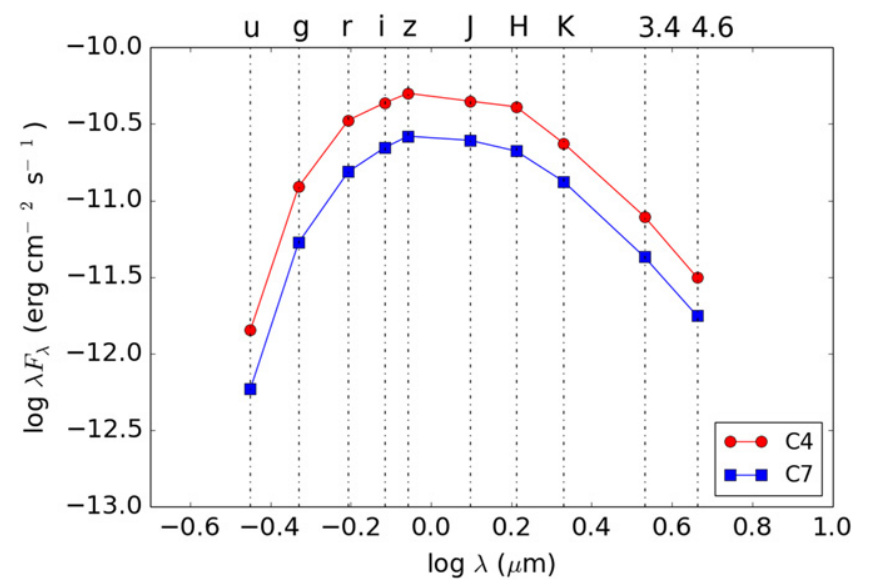

Figure 1. Spectral energy distributions of comparison (C7) and check star (C4). In addition to the $r, i$, and $z$ data from this work, archival data from SDSS, 2MASS, and WISE are adopted.

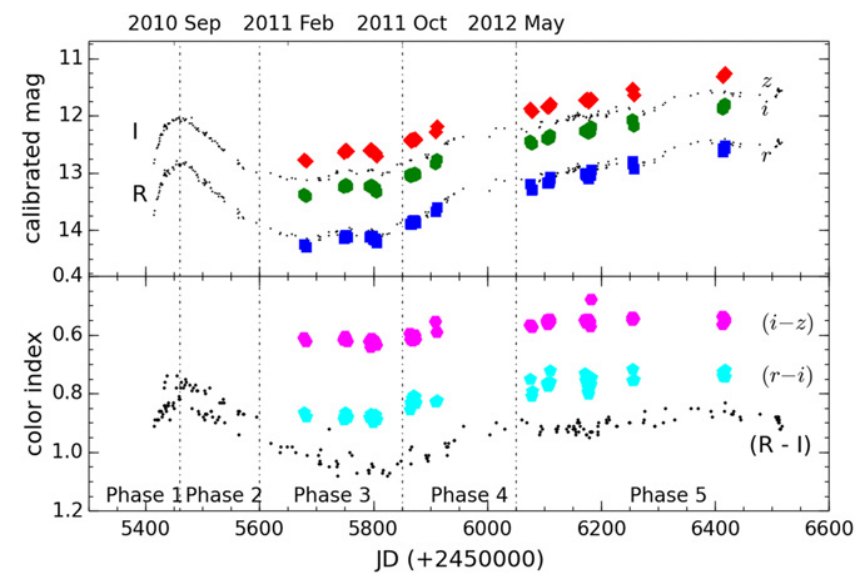

Figure 2. Top: light curves of HBC 722 collected during our observed period (2011 April-2013 May). The upper (red), middle (green), and the the lower (blue) symbols represent light curves of the $z, i$, and $r$ bands, respectively. For comparison, Semkov et al. (2012a, 2014) data of the $I$ and $R$ bands are also plotted as small black dots. Bottom: color variations. The upper (pink) and middle (cyan) dotted curves represent the $i-z$ and $r-i$ color indices, respectively. We also plot the $R-I$ color from Semkov et al. (2012a, 2014) for reference (small black dots). The small offsets between our data and the reference data are a result of the different filter system. The data used to create this figure are available.
Table 3

Classification of Phases

\begin{tabular}{lccll}
\hline \hline Period & Date $($ UT $)$ & JD $(+2450000)$ & Magnitude & Color \\
\hline Phase 1 & -2010 Sep & -5460 & Brighter & Bluer \\
Phase 2 & 2010 Sep-2011 Feb & $5461-5600$ & Fainter & Redder \\
Phase 3 & 2011 Feb-2011 Oct & $5601-5850$ & Constant & Redder \\
Phase 4 & 2011 Oct-2012 May & $5851-6050$ & Brighter & Bluer \\
Phase 5 & 2012 May- & $6051-$ & Brighter & Constant \\
\hline
\end{tabular}

Miller et al. (2011). After dimming by $1.4 \mathrm{mag}(R)$ during six months, the source remained relatively constant in brightness for another few months with only small bounces, but color continuously became redder. We label it as phase 3 , which describes the relatively constant state in the period of outburst. In contrast, in phase 4, HBC 722 started to re-brighten slowly from 2011 October. It steadily recovered its luminosity and the color curves showed distinctive bluer tendency again until 2012 May. Lastly, in phase 5, the brightness continuously increased and went over its first peak of luminosity in 2013 May observation. In contrast to phase 4, color curves of the last phase have weak bluer tendency or close to constant with small fluctuations. According to Semkov et al. (2014), HBC 722 maintains similar brightness from the secondary peak in 2013 May.

We found hints of short-term phenomena distinct from the long-term behaviors. Figure 3 shows sample data collected over two weeks in 2011 August. The upper two panels are the light and color curves of HBC 722, and the lower two panels are those of the comparison star, $\mathrm{C} 7$. We averaged the data of each night to see day scale brightness and color variabilities. Note that this period belongs to phase 3 in the long term, during which HBC 722 stayed relatively constant in brightness and became redder in color (see Figure 2). In contrast, day scale fluctuations are seen with the other short scale decreasing tendency of $0.1 \mathrm{mag}$ amplitude. Color hardly changed at the same time. Figure 4 is another sample data collected in 2012 September. Figure 4 is produced in the same manner as Figure 3. This period is in phase 5 with respect to the long term, where luminosity became brighter and color showed slight changes. As with the previous case, there are small fluctuations in light curves in the short term. In summary, distinct behaviors on different timescales imply that there could be shorter timescale mechanisms in HBC 722 system. 


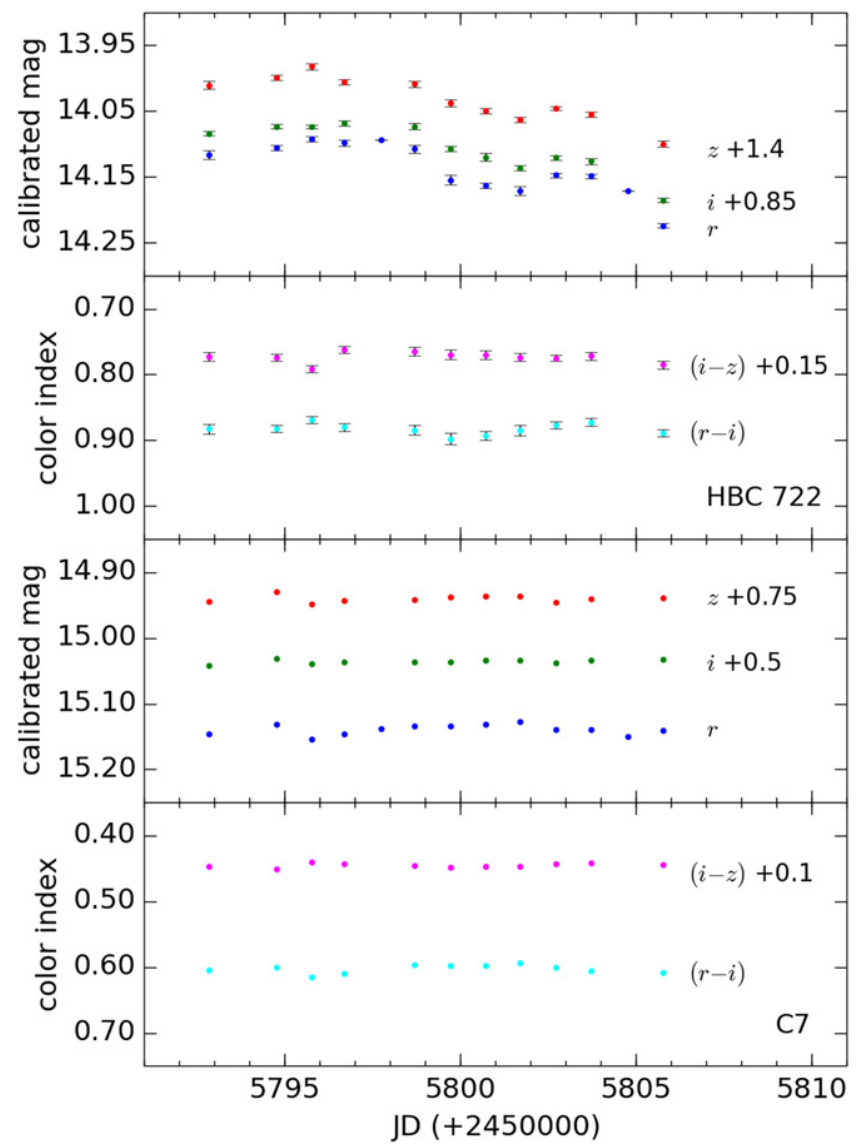

Figure 3. Sample of day scale light and color curves in 2011 August (phase 3). The top two panels are light and color curves of HBC 722 and the bottom two panels are those of comparison star C7. In the light curves, the upper (red), middle (green), and the lower (blue) dots illustrate light curves of $z, i$, and $r$ bands, respectively. In the color curves, upper (pink) and the lower (cyan) dots represent $i-z$ and $r-i$ color, respectively. Data taken in a single night are averaged. For better visibility, offsets are applied. The light curves suggest the presence of day scale variabilities, whereas color hardly changed in day scale in the same period.

\subsection{Color-Magnitude Diagram}

Based on our $r, i$, and $z$ band photometry in phases 3-5, we present $i$ versus $r-i$ and $i-z$ color-magnitude diagrams in Figure 5. In the figure, the upper and lower sequences depict $i-z$ and $r-i$ colors, respectively. Each point is obtained by averaging whole data taken in a night. During the overall observed period, both $r-i$ and $i-z$ colors became bluer as brightness increased. The $r-i$ tendency is slightly steeper than that of $i-z$. This behavior is expected due to the temperature change of the source. When accretion-related variability occurs, the amount of energy from the source and the emission distribution with wavelengths change. This phenomenon results in the variation of SED shape. At that time, the wavelength range shorter than emission peak would lie on the blue edge or "Wien side" of the SED, and thus this part is very sensitive to the temperature change (e.g., Hartmann 2008). In the case of HBC 722, the characteristic wavelengths are located at optical/near-infrared. Thus over the long term, the bluer tendency at optical/near-infrared colors as brightness increases is reasonable. We also analyzed the data in each phase. Color remained constant in phase 3 and became bluer when it entered to phase 4 . In phase $5, i-z$ color remained almost constant again but $r-i$ color became even bluer. Additionally, there were local

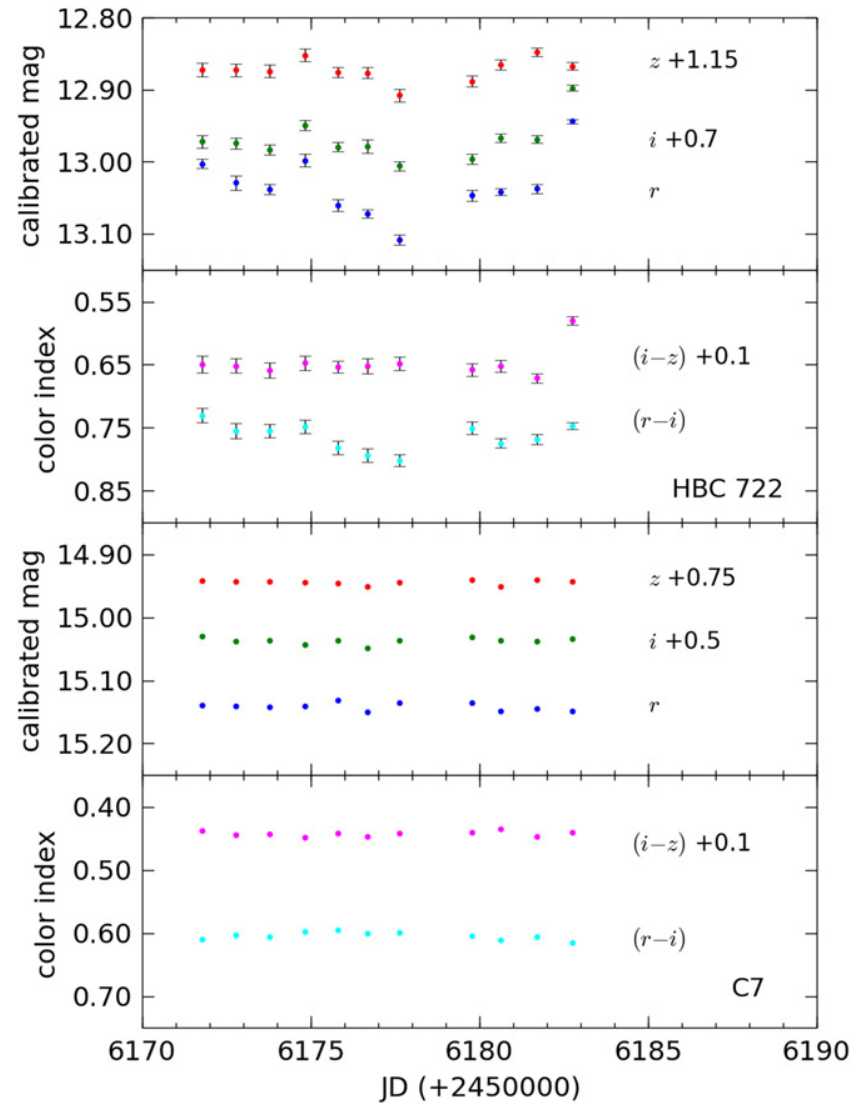

Figure 4. Sample of day scale light and color curves in 2012 September (phase 5); The panels and symbols follow the same description as Figure 3. There is a set of peculiar points on 2012 September 12 (JD 2456182.76). We did not discard these points based on the stable sequence of the comparison stars at the same time as seen in the third panel, although we note that the weather on the night was in non-photometric condition.

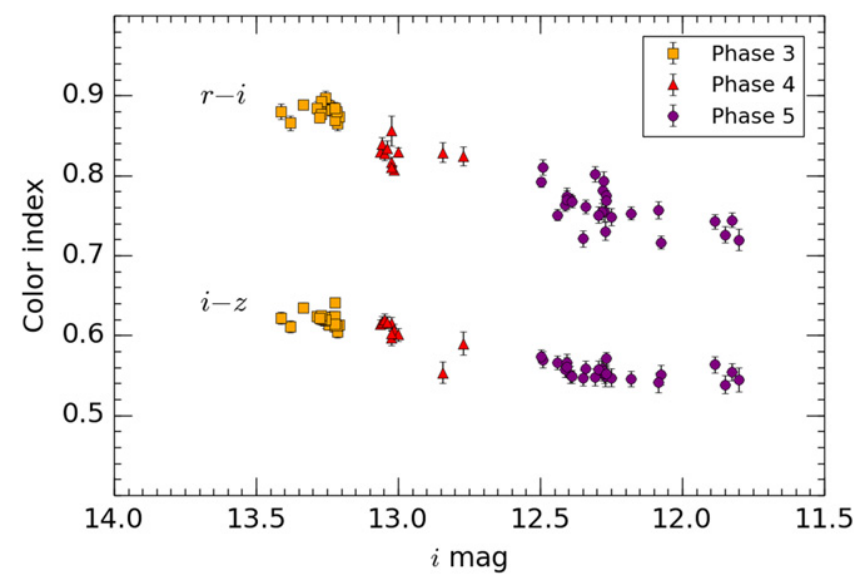

Figure 5. Color-magnitude diagram during the observed period. The upper and lower layers show $i-z$ and $r-i$ color indices, respectively. To look at day scale color variation, the entirety of the data taken in a night are averaged.

fluctuations of a few days in the phases. In the long term, the grouped phases well followed the bluer trend, but in the short term, the order of a few days variation fluctuated within the grouped phases, which is not in the regular sequence of the long-term trend. 


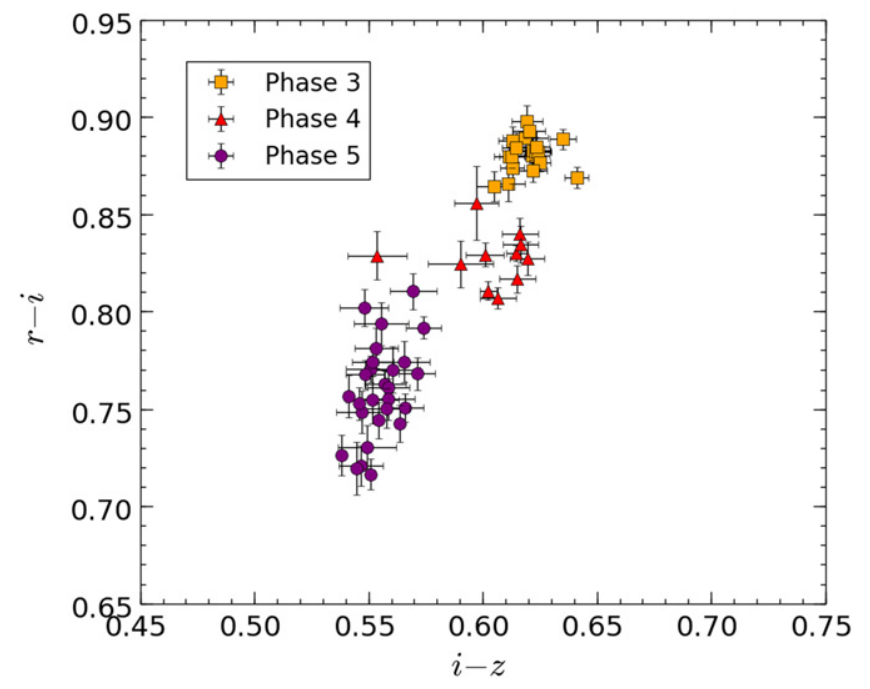

Figure 6. Color-color diagram for observed period. Each dot represents the average value in a night. We can see that the color hardly moved during phase 3 (2011 February-2011 August), it started to become bluer while the source brightened in phase 4 , and returned to constant or less blue status in phase 5 .

\subsection{Color-Color Diagram}

Figure 6 shows a $r-i$ versus $i-z$ color-color diagram of our observational data. Points averaged over a single night are presented to see day scale behaviors. Again, data are grouped according to the phases. We hardly see color variations during phase 3 , but they moved toward the bluer direction with the increase in brightness in phase 4 . It remains in a similar position from phase 5 , in which the color hardly varied in $i-z$ but became bluer in $r-i$. Both Figures 5 and 6 illustrate varying color features of HBC 722 in regard to the phases. Therefore, we argue that these color changes might be caused by altering physical properties in individual phases.

\section{DISCUSSION}

\subsection{Periodic Signals}

Green et al. (2013) reported two families of day scale periodic variability in the SDSS $r$ band (5.8 and 1.28 day, 44 and $16 \mathrm{mmag}$ amplitude, respectively) in the re-brightening phase. The authors proposed two scenarios, assuming the 5.8 and 1.28 day periods were attributed to the stellar rotation period and disk instability and vice versa. Additionally, they suggest flickering rather than a fixed asymmetry at the inner disk edge as an alternative source of the periodicities, even though the families of periodicity continue in a full year timescale. In this section we look for evidence of short-term periodic variability in the $r, i$, and $z$ bands and check the trend with Green et al. (2013).

We first search for periodicities by computing a generalized Lomb-Scargle periodogram (Zechmeister \& Kürster 2009) for the photometry obtained in each band. The normalized power at each frequency $\omega$ is given by

$$
P(\omega)=N_{H} \frac{\chi_{0}^{2}-\chi^{2}(\omega)}{2 \chi_{0}^{2}}=\frac{N_{H}}{2} p(\omega),
$$

where $\chi^{2}(\omega)$ is computed for the best-fitting sinusoidal signal at that period, $\chi_{0}^{2}$ is the weighted mean of the observations, and $N_{H}$ is the number of degrees of freedom. $p=0$ indicates no

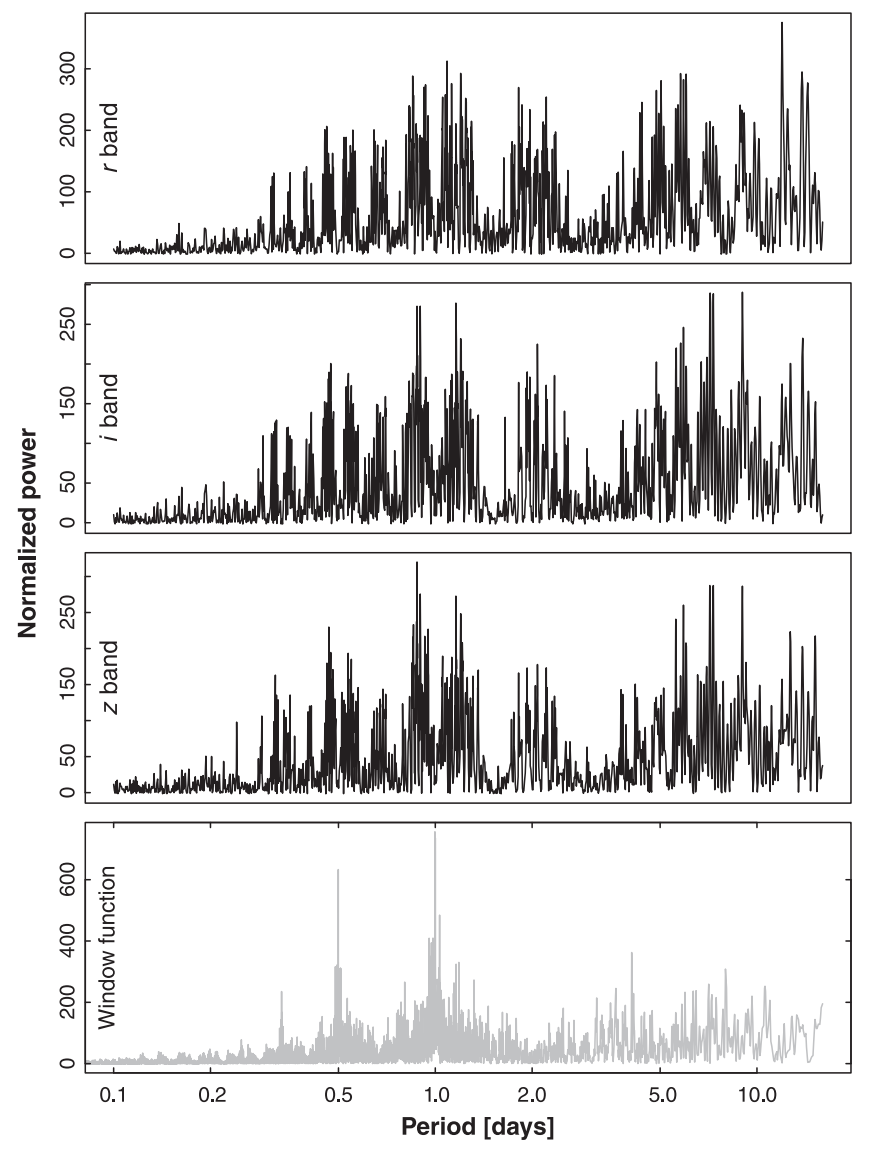

Figure 7. Lomb-Scargle periodogram of the data at each band. The bottom panel shows the periodogram of the window function.

improvement over the null hypothesis (no coherent signal), while $p=1$ is a perfect fit of the data. In order to account for the long-term trend in the brightness of HBC 722 and any drifts in the photometric baseline, we separated each observing run into an independent data set with an adjustable offset. Given that the floating offsets can affect the strength of the signal at each periodicity, we compute the power at each periodicity by self-consistently including the offsets in the minimization of the parameters.

We show the computed periodogram in Figure 7. We only scan for periodicities between 0.1 and 16 days; this range takes into account the periodicities that are effectively sampled by the data. The bottom panel of Figure 7 shows the periodogram of the window function, which indicates the presence of spurious periodicities related to the observational cadence, displaying the usual sharp peak at 1 day (with an alias at 0.5 days).

The periodograms in Figure 7, similar to the data in Green et al. (2013), show an abundance of strong peaks (with very low false alarm probabilities) at a number of periods. The fact that HBC 722 is an active star makes the interpretation of the data in the time domain a difficult task, since it is expected to exhibit high-frequency noise and flickering to a degree. In order to attempt to tentatively identify periodicities over two years, as opposed to the underlying noise, we also characterize signals according to the goodness of the model, as computed by a cross-validation algorithm. Cross-validation algorithms can help identify overfitting or underfitting by resampling the data. We use here the "leave-one-out" implementation that we divide 
Table 4

Best-fit Solutions in each Band

\begin{tabular}{lccrr}
\hline \hline & 1st per. (day) & 2nd per. (day) & $\log \mathcal{L}$ & $\chi^{2}$ \\
\hline$r$ band & & & & \\
\hline No periodicity & $\ldots$ & $\ldots$ & 747.57 & 32.9 \\
One periodicity & $\ldots$ & 11.97 & 431.91 & 17.3 \\
Two periodicities & 11.99 & 7.15 & 293.08 & 10.7 \\
\hline$i$ band & & & & \\
\hline No periodicity & $\ldots$ & $\ldots$ & 434.45 & 16.37 \\
One periodicity & $\ldots$ & 0.95 & 124.57 & 6.68 \\
Two periodicities & 9.03 & & & \\
\hline$z$ band & & $\ldots$ & 24.45 & \\
\hline No periodicity & $\ldots$ & 0.88 & 42.60 & 5.76 \\
One periodicity & $\ldots$ & 0.94 & -46.48 & 4.00 \\
Two periodicities & 9.02 & & & \\
\hline
\end{tabular}

the full data set of $N$ observations into a training set of $N-1$ observations and a testing set of a single observation, rotated among all observations for each band; each training set is used to derive a new fit. The goodness of the fit at each testing data point is then used to compute the combined likelihood $(\log \mathcal{L})$. The combined likelihood is defined as

$$
\log \mathcal{L}=\sum_{i}^{N} \log \left(\frac{y_{i}-f_{i}\left(\theta_{i}\right)}{\sigma_{i}}\right)
$$

where $y_{i}$ is the $i$ th observation comprising the testing set, $f_{i}\left(\theta_{i}\right)$ is the corresponding prediction from the model with the set of parameters $\theta_{i}$ derived by fitting the training set, and $\sigma_{i}$ is the corresponding uncertainty. A fit that has lower $\log \mathcal{L}$ compared with an alternative fit has a higher predictive power.

To facilitate comparison with the results of Green et al. (2013), we derive a set of potential two-signal solutions using a grid search, which starts with the periodogram computed for the single frequency search (Figure 7), then computes a periodogram for the residuals and adds candidate second signals to the fit.

Table 4 shows the best-fitting solutions for each band. Since each band probes potentially different physical phenomena, we did not combine the fits and instead computed each solution separately for each band. For each solution, we list the period (s), normalized $\chi^{2}$ and, likelihood computed by the crossvalidation algorithm $(\log \mathcal{L})$. First, we note that according to the $\log \mathcal{L}$ metric, two-signal solutions are strict, marked improvements over one-signal solutions and no-signal solutions; this suggests that the models should not be overfitting the data, or be driven by small features in the photometry. Second, we note that there are three families of signals-around $\sim 6$, $\sim 10$, and $\sim 1$ days. This is broadly consistent with the work of Green et al. (2013), which uncovered similar families of periodicities. Unfortunately, given the noisiness of the data in the frequency domain and the potential for aliasing, it is hard in practice to disentangle the different periodicities exhibited by the data.

Figure 8 shows the signal harmonics and the phased data for each band. We note there is substantial scatter both within and among the different observation runs, even after the two signals with the largest amplitude are removed. The residual scatter is likely due to high-frequency noise, in accordance to the nature of HBC 722.

\subsection{Spectral Energy Distribution}

To look at the deviations of spectral emissions in the postoutburst phase, we plot the multiple SEDs for several different brightness states in Figure 9. We take the date of Semkov et al. (2012b, 2014) for the $B, V, R$, and $I$ bands from the outburst of 2010-2013 May. Data in the $r, i$, and $z$ bands are taken from this work. For near-infrared, we take $J, H$ and $K_{S}$ band data from Kóspál et al. (2011), Antoniucci et al. (2013), and Sung et al. (2013). All literature data are converted from the Vega magnitude to $\mathrm{AB}$ magnitude system by using the formula of Blanton \& Roweis (2007). We match the archival data to our $r$, $i$, and $z$ data to construct SEDs with adjacent nights. The first SED is from the first peak in 2010, on the boundary between phase 1 and phase 2 in our classification. We only use literature data taken in 2010 September 19 and 20. The second one represents phase 3, which shows relatively calm state, constructed by the data of 2011 April 28, 30 and May 2. The third one is on the boundary between phase 3 and phase 4, when HBC 722 started to re-brighten. We use 2011 October 30 data for all wavelengths. The fourth one is on the boundary between phase 4 and phase 5 , when the source reached to similar brightness with the first peak brightness in 2010 . We use 2012 May 20 and 27 data for this state. The last one is from phase 5 when brightness exceeded the first peak. We use the data of 2013 April 14 and May 4 and 5.

To begin with, there is a main difference between the SED of re-brightening period and that of the first peak. The shape of SED for re-brightening period shows a redder feature than that of first peak, even at phase 5, when the brightness surpassed the first peak. Therefore comparing with the original flaring, HBC 722 showed a greater increase in brightness but less blue in color at re-brightening state.

From phase 3 to phase 5, the shape of the SED also changed slightly. The gradient became less steep as HBC 722 rebrightened, which suggests that the emission from shorter wavelength becomes higher. Thus in the long term, the source became bluer. This is consistent with our color analysis in the previous section. Johnstone et al. (2013) proved the time evolution of the SED in outburst with their established model. When the accretion rate increases, the peak of SED moves toward a shorter wavelength, which fits for a higher temperature blackbody. Also, they predicted that the first indication of heating is an increase in luminosity of the source at a nearer or shorter wavelength region than the peak of the SED. Our time evolution of the SED in the re-brightening state shows a good agreement with their prediction.

Assuming that the emissions entirely came from disk accretion, we calculate relative accretion rate as a function of time. The bolometric luminosities limited at optical-nearinfrared are obtained in each phase (the same epochs of the SEDs). By taking the accretion luminosity formula and properties of HBC 722 used in Green et al. (2013), accretion rates are estimated. We normalize the values for the minimum brightness epoch of phase 3 (2011 April) and present relative accretion rate change (Figure 10). The actual accretion rate obtained at the similar epoch to phase 3 reported in Green et al. (2013) is $\dot{M}=1.31 \times 10^{-6} M_{\odot}$ year $^{-1}$. 

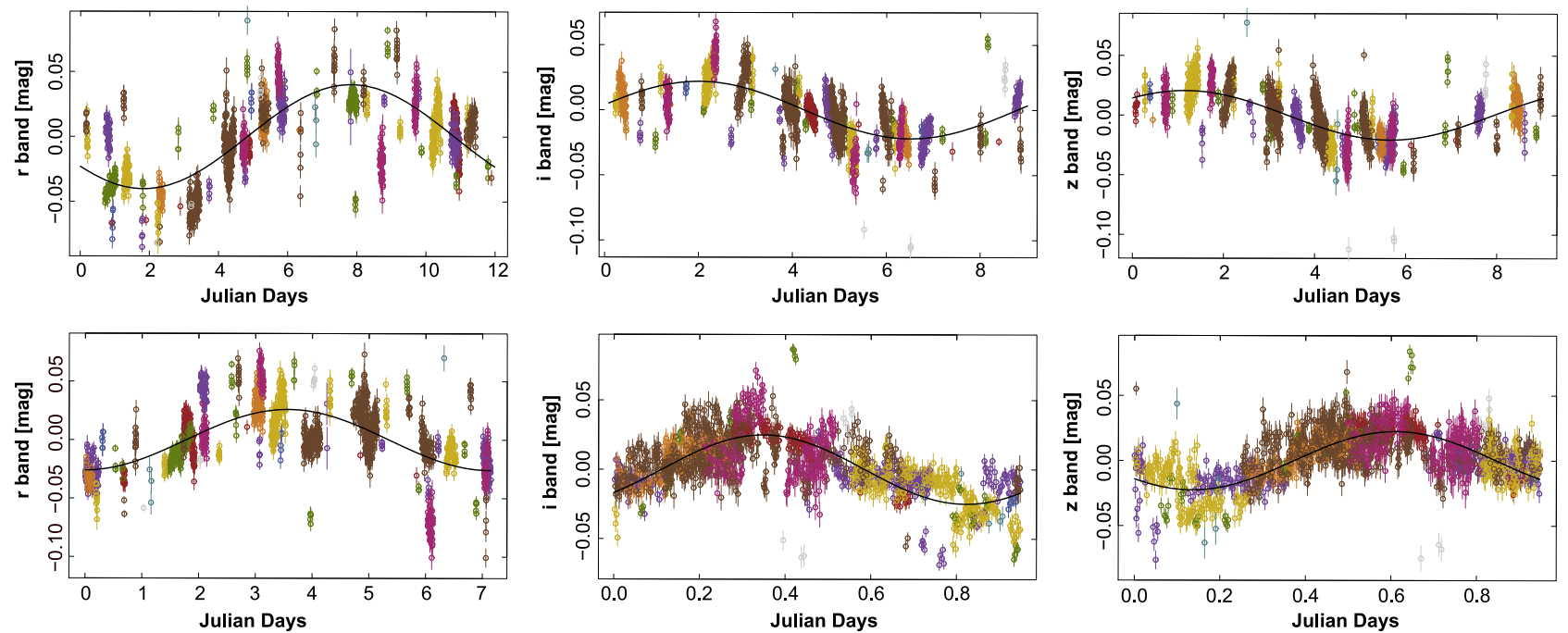

Figure 8. Phased best-fitting models for the data obtained in the $r, i$, and $z$ bands. Each observing run that is shifted with a floating offset is marked with a different color. Note that alternative models are also possible (Table 4).

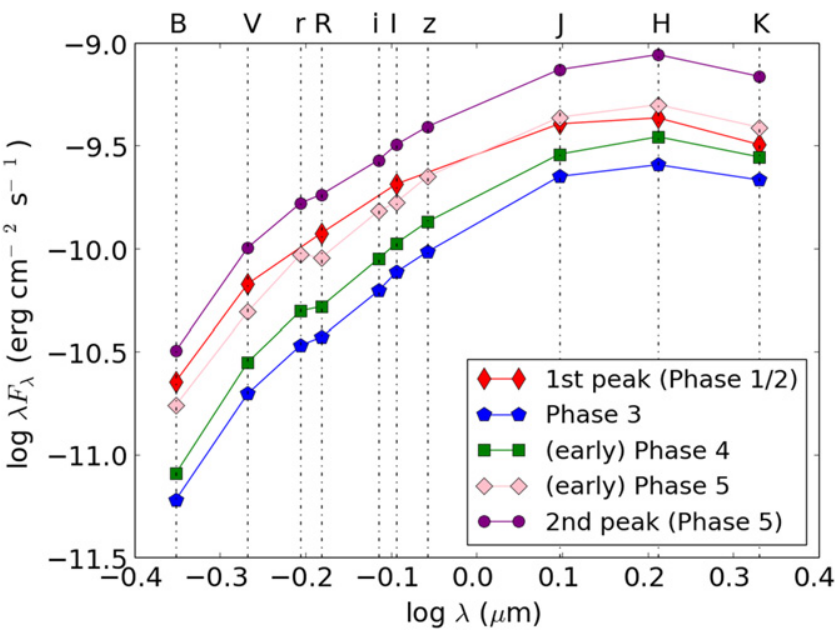

Figure 9. Multi-epoch SEDs of $\mathrm{HBC} 722$ after outburst. The $B, V, R$, and $I$ bands are from Semkov et al. $(2012 \mathrm{~b}, 2014)$. The $r, i$, and $z$ bands are taken from this work. The $J, H$, and $K_{S}$ data are taken from Kóspál et al. (2011), Antoniucci et al. (2013), and Sung et al. (2013). All data except $r, i$, and $z$ are converted from the Vega to $\mathrm{AB}$ magnitudes system using Blanton \& Roweis (2007).

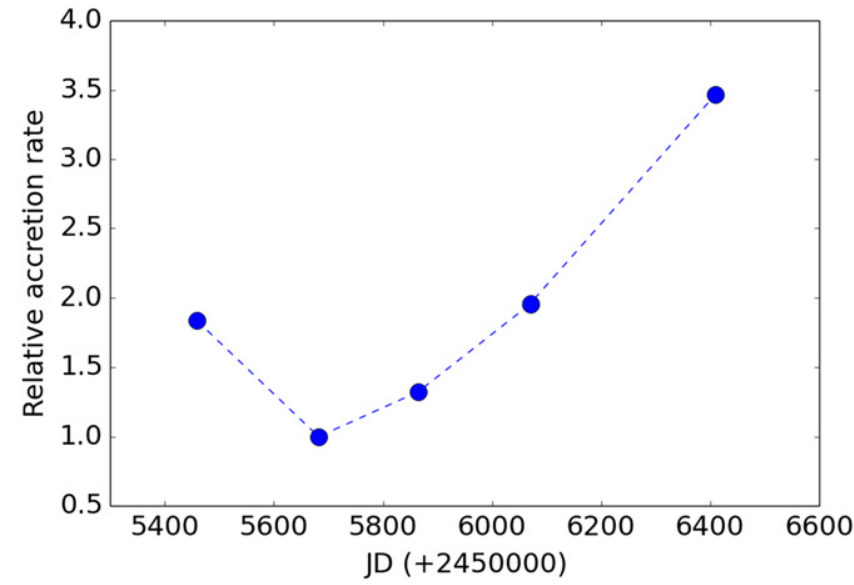

Figure 10. Relative accretion rate in each phase. We normalize derived accretion rate values to the minimum brightness epoch after outburst $(2011$ April) to compare the change of accretion rate in the post outburst phase.

\subsection{Flickering}

Although only small number of outbursting YSOs have been detected, these uniquely enhanced systems provide insight on how the accretion process occurs from the innermost part of circumstellar disk to the central star, which plays an important role for understanding the evolution of YSOs.

In accordance with Kenyon et al. (2000 and references therein.), "flickering" is randomly fluctuated small amplitude (0.01-1.0 mag) variations in dynamic timescales. It could be observed in cataclysmic variables and erupting YSOs. Flickering is often thought to be a signature of disk accretion. The most widely accepted origin of flickering is a temperature region between the central star and disk, which is in the vicinity of inner edge of the disk. Since a large amount of disk material falls to the stellar surface from the inner disk, flickering could be evidence for inhomogeneous accretion flow (e.g., Shu et al. 1994; Bastien et al. 2011).

In order to check short-term behaviors of intra-day and day time scales, we quantify the potential variabilities of HBC 722 in the $r, i$, and $z$ bands. We expect to see footprints of flickering by using a micro-variability method developed by Jang \& Miller (1997).

$$
\begin{aligned}
C_{1} & =\frac{\sigma(\text { Object }- \text { Comp } 1)}{\sigma(\text { Comp } 1-\text { Comp2 })} \text { and } \\
C_{2} & =\frac{\sigma(\text { Object }- \text { Comp } 2)}{\sigma(\text { Comp } 1-\text { Comp } 2)}
\end{aligned}
$$

$C_{1}$ is calculated by standard deviation of differential photometry for an object (Object) and a comparison star (Comp1), divided by that of the comparison star (Comp1) and a check star (Comp2). $C_{2}$ is also calculated in the same manner. Finally we derived the parameter $\mathrm{C}$ by taking the average of $C_{1}$ and $C_{2}$ (e.g., Romero et al. 1999; Gupta et al. 2008). Since the calculation of parameter C includes possible variations of the comparison star, a valid result for variability of HBC 722 can be obtained only if the variation of HBC 722 is superior to that of comparisons. According to Jang \& Miller (1997), parameter C depends on normal distribution, so we can suggest that potential variabilities exist with $90 \%$, $95 \%$ and $99 \%$ confidence level if $\mathrm{C}$ values are $1.64,1.96$, and 
Table 5

Result of Day Scale Variability of HBC 722

\begin{tabular}{lccccc}
\hline \hline Month & Filter & Duration (day) & $\mathrm{C}_{r}$ & $\mathrm{C}_{i}$ & $\mathrm{C}_{z}$ \\
\hline $2011-07$ & $r, i, z$ & 6 & 2.54 & 2.73 & 1.83 \\
$2011-08$ & $r, i, z$ & 13 & 4.86 & 6.63 & 4.69 \\
$2011-11$ & $r, i, z$ & 9 & 2.44 & 2.42 & 1.59 \\
$2012-06$ & $r, i, z$ & 7 & 4.97 & 4.34 & 3.29 \\
$2012-09$ & $r, i, z$ & 11 & 2.68 & 1.98 & 1.39 \\
$2013-05$ & $r, i, z$ & 4 & 3.39 & 3.93 & 2.83 \\
\hline
\end{tabular}

Note. C values represent relative brightness derivation of HBC 722 to that of comparison star. It provides statistical confidences of variability of the source. $\mathrm{C}$ value above $1.64,1.96$, and 2.57 infer confidence level of the variability in $90 \%, 95 \%$, and $99 \%$ respectively.

2.57, respectively. Finally, we substitute $\mathrm{HBC} 722, \mathrm{C} 7$, and C4 for Object, Comp1, and Comp2, respectively.

The results of day scale variability of HBC 722 are presented in Table 5 and Figure 11. We do not include 2011 April, 2011 December, 2012 May, and 2012 November runs because each covered only a few nights including non-photometric nights. For the majority of nights, $\mathrm{C}$ values are over 2.57 , which implies HBC 722 shows variability with a $99 \%$ confidence level in the day scale. On the other hand, $\mathrm{C}_{r}$ in $2011 \mathrm{July}, \mathrm{C}_{r}$ and $\mathrm{C}_{i}$ in 2011 November, and $\mathrm{C}_{i}$ in 2012 September have values between 1.96 and 2.57. According to the aforementioned rule, these have $95 \%$ confidence for their variabilities. Lastly, $C_{z}$ in 2011 July is located between 1.64 and 1.96, which is at a slightly lower confidence level than the others, but still $90 \%$ confidence of variability. To summarize, HBC 722 strongly shows day scale variability in 2011 August, 2012 June, and 2013 May. It displays meaningful variabilities in the other months as well. Therefore, we conclude that HBC 722 is flickering. Since there is no certain relation between the length of observation and amplitude of brightness change, it is unlikely that the duration of continuous observation affects the $\mathrm{C}$ values.

According to the historical efforts to find clues of flickering in the outburst stage of cataclysmic YSOs, many studies focused on finding day scale periodic and aperiodic variations. However, using the properties of low mass YSOs and their Keplerian disks, we can explore IDV, which could originate from between the innermost part of the disk and the central star. Opportunely, our observing time at the re-brightening state of HBC 722 is related to the re-stimulated disk accretion activity. Because of our short cadence monitoring observation strategy, we can also look at the behaviors of HBC 722 in the intra day scale. We quantify the IDV in the same manner with the day scale variability. The results of derived $\mathrm{C}$ values are tabulated in Table 6.

In the analysis of micro-variability of each night, we find that $\mathrm{C}_{r}$ and $\mathrm{C}_{i}$ on 2011 July $5, \mathrm{C}_{i}$ on 2012 September 2 and 2013 May 1 have between 1.96 and 2.57, which means potential variabilities with a $95 \%$ confidence level. Additionally, $\mathrm{C}_{r}$ on 2011 August 24, 2011 November 4, 2012 September 6, and 2013 May 5 are all between 1.64 and 1.96, which implies $90 \%$ confidence of potential variability. Meanwhile, many of the $\mathrm{C}$ values in $z$ band, and a few of $r$ and $i$ bands are lower than 1.0. Since the micro-variability method depends on the comparison and check star, these values can be caused by the brightness differences among the object (HBC 722), comparison (C7), and check stars (C4). In this analysis, IDV is less convincing than day scale variability. Note that we suggest statistical values for variability rather than specific values.

There are several previous studies of short-term variability in FUors. Herbig (2003) observed FU Orionis and revealed $\sim 14$ days of spectroscopic periodicity in $\mathrm{P}$ Cygni profiles, especially in $\mathrm{H} \alpha$, lasting more than 1.5 years. The author also discovered another 3.54 days of periodic variation arising from the inner structure of the photosphere. Powell et al. (2012) confirmed the periodicities found by Herbig (2003) and suggested that the periodic phenomena continued over 10 years. Recently Siwak et al. (2013) discovered 2-9 day quasiperiodic features in FU Orionis using the Microvariability and Oscillations of Stars satellite. Furthermore, Siwak et al. (2013) stated that the features could be caused by a dump of plasma, or magneto-rotationally unstable heterogeneities in the localized accretion disk rotating at different Keplerian radii. Meanwhile, Clarke et al. (2005) reported day scale non-periodic fluctuations in another classical FUors. They detected photometric variabilities with amplitudes of 0.1 and $0.3 \mathrm{mag}(V)$ for V1057 Cyg and V1515 Cyg, respectively, which might be caused by flickering events.

In HBC 722, day scale non-periodic variabilities similar to the V1057 Cyg and V1515 Cyg cases are detected. Because two classical FUors are brighter than HBC 722 in the optical, it is plausible that HBC 722 is flickering with a smaller amplitude. We found the variabilities last more than two years with diverse amplitudes.

Looking back to other studies of IDV for flaring YSOs, Kenyon et al. (2000) collected photometric data of FU Orionis and detected random brightness fluctuations in a dynamic timescale of a day or less, with $0.035-0.1 \mathrm{mag}$ amplitude $(V)$. Bastien et al. (2011) conducted rapid cadence time series photometry for V1647 Orionis in the outburst stage, which belongs to another category of eruptive YSO, EXors. As a result, 0.13 day (51 mmag amplitude) periodic variability was found. They believed that the periodic variability would be related to flickering by detecting "flickering noise" signs in the power spectrum of the light curves. Since HBC 722 still remains enhanced, further investigation with sufficient data will more clearly reveal IDV and properties of intra-day scale flickering.

In Figure 11, we attempt to find a relation between the longterm brightness change and the short-term variability. Two of the $\mathrm{C}$ values around 2011 August and 2012 September are particularly large, and they lie at the boundary between the phase lines (noted in Table 3). These larger points near the boundary of phases might be caused by a transition to the next phases, as one possible interpretation. It can be proved if additional short-term variabilities are detected when the longterm brightness tendency changes. Finally, we find a tentative relation that $\mathrm{C}$ values get lower from $r$ to $z$, implying smaller amplitude of variation in longer wavelength. A few explanations might be possible. First, this tendency can be simply due to the brightness effect. Under the assumption of no intrinsic variabilities for two objects, a brighter star shows a smaller brightness deviation than a dimmer one. In our case, HBC 722 clearly shows intrinsic variability but this brightness effect would be added. In other words, in the $r, i$, and $z$ bands, HBC 722 is brighter than comparison stars but the brightness difference in the $z$ band is greater than that in the $r$ band. Since 
Table 6

Result of Intra-day Variability (IDV) of HBC 722

\begin{tabular}{|c|c|c|c|c|c|c|}
\hline Date (UT) & Filter & Number of Frames & Observing Time (hour) & $\mathrm{C}_{r}$ & $\mathrm{C}_{i}$ & $\mathrm{C}_{z}$ \\
\hline 2011-04-26 & $r, i, z$ & 24 & 0.5 & 1.10 & 0.80 & 1.19 \\
\hline 2011-07-05 & $r, i, z$ & 311 & 4.5 & 2.11 & 2.04 & 1.48 \\
\hline 2011-07-11 & $r, i, z$ & 47 & 0.9 & 1.16 & 1.47 & 1.04 \\
\hline 2011-08-31 & $r$ & 791 & 5.7 & 1.43 & $\ldots$ & $\ldots$ \\
\hline 2011-11-02 & $r, i, z$ & 211 & 5.3 & 1.50 & 1.08 & 0.82 \\
\hline 2011-11-04 & $r, i, z$ & 116 & 2.9 & 1.71 & 1.24 & 0.98 \\
\hline $2012-05-30$ & $r, i, z$ & 300 & 3.8 & 1.45 & 0.96 & 0.90 \\
\hline 2012-06-28 & $r, i, z$ & 399 & 6.2 & 1.42 & 0.88 & 0.96 \\
\hline 2012-07-01 & $r, i, z$ & 182 & 4.0 & 1.45 & 1.05 & 0.88 \\
\hline 2012-07-02 & $r, i, z$ & 32 & 0.5 & 1.06 & 1.24 & 0.94 \\
\hline 2012-09-01 & $r, i, z$ & 328 & 6.2 & 1.07 & 1.22 & 1.18 \\
\hline 2012-09-02 & $r, i, z$ & 492 & 7.1 & 0.97 & 2.29 & 1.01 \\
\hline $2012-11-26$ & $r, i, z$ & 53 & 0.5 & 0.93 & 1.06 & 1.00 \\
\hline 2013-04-30 & $r, i, z$ & 162 & 2.2 & 0.99 & 1.10 & 0.86 \\
\hline 2013-05-01 & $r, i, z$ & 144 & 1.7 & 1.24 & 2.20 & 0.98 \\
\hline 2013-05-04 & $r, i, z$ & 143 & 2.2 & 0.99 & 1.03 & 0.80 \\
\hline $2013-05-05$ & $r, i, z$ & 150 & 1.8 & 1.68 & 1.70 & 0.76 \\
\hline
\end{tabular}

Note. C values for the confidence level follow the same rule in Table 5.

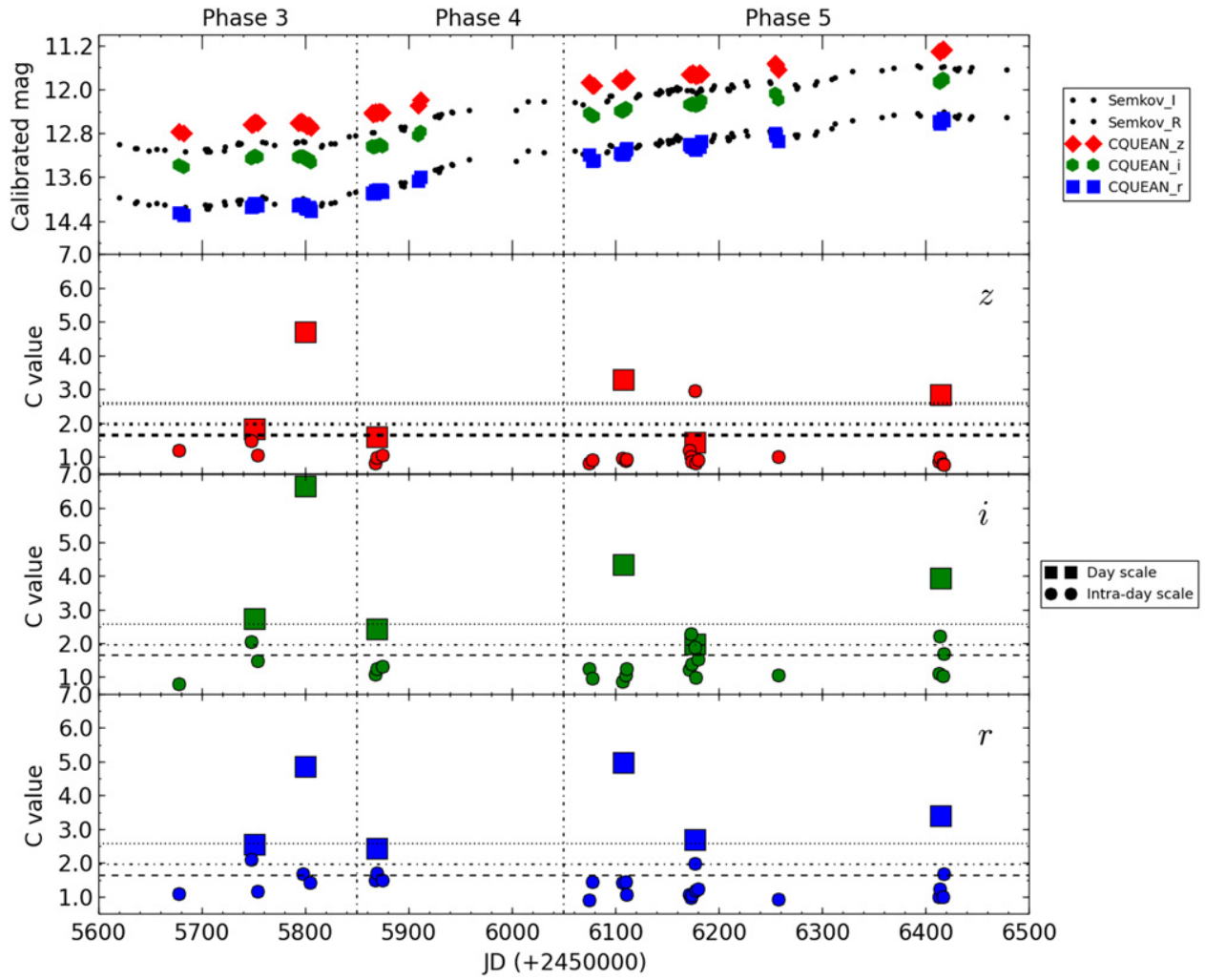

Figure 11. Overall light curves and plots of $C$ values. The top plot shows light curves from 2011 April to 2013 May as a reference for long-term brightness variation. The upper (red), middle (green), and lower (blue) curves depict the $z, i$, and $r$ bands; the $I$ and $R$ bands references are also presented(black). The second to the fourth plots show $\mathrm{C}$ value distribution for the $z, i$, and $r$ bands, respectively. Large squares and small circles indicate day scale from Table 5 and intra-day scale from Table 6 , respectively. 
the $\mathrm{C}$ values that we used are obtained by relative amount of brightness deviation from that of comparisons, the values could be affected by brightness difference in each band. Therefore the $\mathrm{C}$ values could decrease with increasing wavelengths. Second, intrinsic characteristics of HBC 722 could appear. Sung et al. (2013) reported that HBC 722 showed strong correlation between flux variation and fading period after outburst. They estimated the flux variations from maximum brightness in 2010 to minimum in 2011 and compared them with fading periods. As a result, both flux variation and fading timescale are larger at shorter wavelengths. The flux variation tends to decrease linearly as wavelength increases in the optical/near-infrared range. Therefore we could expect that there is an inverse proportion relation between wavelengths $(r, i$, and $z$ bands $)$ and amplitudes.

\section{CONCLUSION}

We observed HBC 722 in the SDSS $r, i$, and $z$ bands from 2011 April to 2013 May with CQUEAN attached to the $2.1 \mathrm{~m}$ Otto Struve telescope at McDonald Observatory. The photometric results show that HBC 722 re-brightened for two years and presented unprecedented high brightness. The color also became bluer during the same period. However, the brightness and color occasionally maintained constant status rather than steadily changing, which could be related to physical processes in the inner disk. Thus we divide the post-outburst period into five phases according to brightness and color variations. We analyze color-magnitude diagrams and color-color diagrams to depict the tendency along with the phases and possible day scale variabilities. Different shapes of optical/near-infrared emissions between the first peak and henceforward are shown in SEDs. Additionally, multi-epoch SED shapes indicate that HBC 722 became hotter as brightness increased.

We conducted a periodicity check for HBC 722. As shown in Figure 7, HBC 722 exhibits high-frequency noise and flickering, as expected because it is in an enhanced state. Thus we statistically show best-fitting solutions for each band, which were broadly converged in three families of signals around $\sim 6$, $\sim 10$, and $\sim 1$ days. These results are well matched with the discovered periods of Green et al. (2013).

We also investigate short-term variability separated from long-term variations to find indications of flickering. Intra-day and day scale variabilities are quantified by using a microvariability method, which uses the variability of HBC 722 relative to that of comparison star. We find clear evidence of day scale variabilities and weaker signs of IDV in the $r, i$, and $z$ bands. Comparing these short-term variabilities with long-term brightness variations from phase 3 to phase 5 , derived $C$ values of variability tend to be larger near the boundaries of phases. We suggest that there could be transitions of physical processes at the innermost part of disk can be attributed to the change of brightness and color behaviors.
This work was supported by the National Research Foundation of Korea (NRF) grant, No. 2008-0060544, funded by the Korea government (MSIP). The authors thank Prof. Sang-Gak Lee and Prof. Tae Seog Yoon for valuable discussions and suggestions. This paper includes data taken at The McDonald Observatory of The University of Texas at Austin. This research has made use of the USNOFS Image and Catalogue Archive operated by the United States Naval Observatory, Flagstaff Station (http://nofs.navy.mil/data/ fchpix/).

\section{REFERENCES}

Antoniucci, S., Arkharov, A., Klimanov, S., et al. 2013, ATel, 5023, 1

Audard, M., Ábrahám, P., Dunham, M. M., et al. 2014, in Protostars and Planets VI, Vol. 1401, ed. H. Beuther, R. Klessen, H. Dullemond, \& Th. Henning (Univ. Arizona Press), 3368

Bastien, F. A., Stassun, K. G., \& Weintraub, D. A. 2011, AJ, 142, 141

Bell, K. R., \& Lin, D. N. C. 1994, ApJ, 427, 987

Bertin, E., \& Arnouts, S. 1996, A\&AS, 117, 393

Blanton, M. R., \& Roweis, S. 2007, AJ, 133, 734

Clarke, C., Lodato, G., Melnikov, S. Y., \& Ibrahimov, M. A. 2005, MNRAS, 361,942

Cohen, M., \& Kuhi, L. V. 1979, ApJS, 41, 743

Fukugita, M., Ichikawa, T., Gunn, J. E., et al. 1996, AJ, 111, 1748

Goodrich, R. W. 1987, PASP, 99, 116

Green, J. D., Robertson, P., Baek, G., et al. 2013, ApJ, 764, 22

Gupta, A. C., Cha, S.-M., Lee, S., et al. 2008, AJ, 136, 2359

Hartmann, L. 2008, Accretion Processes in Star Formation, Vol. 47 (2nd ed.; Cambridge: Cambridge Univ. Press)

Hartmann, L., \& Kenyon, S. J. 1996, ARA\&A, 34, 207

Herbig, G. H., Petrov, P. P., \& Duemmler, R. 2003, ApJ, 595, 384

Jang, M., \& Miller, H. R. 1997, AJ, 114, 565

Johnstone, D., Hendricks, B., Herczeg, G., \& Bruderer, S. 2013, ApJ, 765,133

Kenyon, S. J., Kolotilov, E. A., Ibragimov, M. A., \& Mattei, J. A. 2000, ApJ, 531, 1028

Kim, E., Park, W.-K., Jeong, H., et al. 2011, JKAS, 44, 115

Kóspál, Á, Ábrahám, P., Acosta-Pulido, J. A., et al. 2011, A\&A, 527, 133

Laugalys, V., Straižys, V., Vrba, F. J., et al. 2006, BaltA, 15, 483

Lee, J.-E., Kang, W., Lee, S.-G., et al. 2011, JKAS, 44, 67

Lim, J., Chang, S., Pak, S., et al. 2013, JKAS, 46, 161

Miller, A. A., Hillenbrand, L. A., Covey, K. R., et al. 2011, ApJ, 730, 80

Park, W.-K., Pak, S., Im, M., et al. 2012, PASP, 124, 839

Powell, S. L., Irwin, M., Bouvier, J., \& Clarke, C. J. 2012, MNRAS, 426, 3315

Romero, G. E., Cellone, S. A., \& Combi, J. A. 1999, A\&AS, 135, 477

Semkov, E. H., Peneva, S. P., Munari, U., et al. 2010, A\&A, 523, 3

Semkov, E. H., Peneva, S. P., Munari, U., et al. 2012, yCat, 354, 29043

Semkov, E. H., Peneva, S. P., Munari, U., et al. 2012, A\&A, 542, 43

Semkov, E. H., Peneva, S. P., Ibryamov, S. I., \& Dimitrov, D. P 2014, BlgAJ, 20, 59

Shu, F., Najita, J., Ostriker, E., et al. 1994, ApJ, 429, 781

Siwak, M., Rucinski, S. M., Matthews, J. M., et al. 2013, MNRAS, 432, 194

Skrutskie, M. F., Cutri, R. M., Stiening, R., et al. 2006, AJ, 131, 1163

Smith, J. A., Tucker, D. L., \& Kent, S. 2002, AJ, 123, 2121

Sung, H.-I., Park, W.-K., Yang, Y., et al. 2013, JKAS, 46, 253

Weintraub, D. A., Sandell, G., \& Duncan, W. D. 1991, ApJ, 382, 270

Wright, E. L., Eisenhardt, P. R. M., Mainzer, A. K., et al. 2010, AJ, 140, 1868

Zechmeister, M., \& Kürster, M. 2009, A\&A, 496, 577 